\title{
Culicinae (Diptera, Culicidae) ocorrentes no Estado do Rio Grande do Sul, Brasil
}

\author{
Jader da Cruz Cardoso ${ }^{1}$, Elio Corseuil ${ }^{2}$ \& José Maria Soares Barata ${ }^{3}$
}

\begin{abstract}
'Laboratório Central de Saúde Pública do Estado do Rio Grande do Sul, Fundação Estadual de Produção e Pesquisa em Saúde, LACEN-RS/FEPPS. 90610-000 Porto Alegre-RS, Brasil. Bolsista CNPq. E-mail: jader@fepps.rs.gov.br

${ }^{2}$ Laboratório de Entomologia, Departamento de Biologia, Faculdade de Biociências, PUCRS. Caixa Postal 1429, $90619-900$ Porto Alegre-RS, Brasil. E-mail: corseuil@pucrs.br

${ }^{3}$ Laboratório de Triatomíneos e Culicídeos, Departamento de Epidemiologia, Faculdade de Saúde Pública, USP. 01246-904 São Paulo-SP, Brasil. E-mail: jmsbarat@usp.br
\end{abstract}

\begin{abstract}
Culicinae (Diptera, Culicidae) occurring in the State of Rio Grande do Sul, Brazil. This work lists the species of Culicinae recorded in the State of Rio Grande do Sul, Brazil. The present data is based on adult collections carried out in 11 physiographic zones from September/2001 to August/ 2002, published literature and entomological collection records. Fifty-seven species are listed, including 22 new occurrences to Rio Grande do Sul. The present study increased the geographic distribution range of Aedeomyia squamipennis, Culex chidesteri, Culex quinquefasciatus, Haemagogus leucocelaenus, Mansonia titillans, Ochlerotatus albifasciatus, Ochlerotatus crinifer, Ochlerotatus fluviatilis, Ochlerotatus scapularis, Ochlerotatus serratus, Psorophora confinnis, Psorophora ferox, Sabethes aurescens, Trichoprosopon pallidiventer and Uranotaenia lowii.
\end{abstract}

KEYWORDS. Brazil, Culicinae, distribution, Rio Grande do Sul.

RESUMO. Culicinae (Diptera, Culicidae) ocorrentes no Estado do Rio Grande do Sul, Brasil. Este trabalho lista as espécies e aponta a ocorrência de Culicinae, com base em coletas de adultos realizadas no período de setembro de 2001 a agosto de 2002, em 11 zonas fisiográficas do Estado, busca de informações bibliográficas e verificação de exemplares em coleções. São relacionadas 57 espécies, sendo que 22 constituem novas ocorrências para o Rio Grande do Sul. São ampliados os locais de ocorrência de Aedeomyia squamipennis, Culex chidesteri, Culex quinquefasciatus, Haemagogus leucocelaenus, Mansonia titillans, Ochlerotatus albifasciatus, Ochlerotatus crinifer, Ochlerotatus fluviatilis, Ochlerotatus scapularis, Ochlerotatus serratus, Psorophora confinnis, Psorophora ferox, Sabethes aurescens, Trichoprosopon pallidiventer e Uranotaenia lowii.

PALAVRAS-CHAVE. Culicinae, distribuição geográfica, mosquitos, Rio Grande do Sul.

Os culicíneos são mosquitos de grande interesse para a saúde pública, pois reúnem espécies vetoras de arbovírus, como o da dengue e da febre amarela, além de causar grande incômodo às populações humanas, devido aos hábitos hematófagos das fêmeas (Forattini 2002).

Culicinae constitui a maior subfamília de Culicidae, abrangendo cerca de 3000 espécies (Consoli \& Lourenço-deOliveira 1994), distribuídas em 11 tribos, constituídas por 38 gêneros, sendo 34, dentre os relacionados por Harbach \& Kitching (1998) e quatro, em função dos trabalhos de Reinert (1999), Harbach \& Peyton (2000) e Reinert (2000 a,b). O táxon Phoniomyia, citado por Harbach \& Kitching (1998) como gênero, foi considerado por Judd (1998) como subgênero de Wyeomyia. A Região Neotropical conta com 908 espécies (Papavero \& Guimarães 2000), inseridas em 22 gêneros e 9 tribos (Forattini 2002). Guimarães (1997) registra, para o Brasil, 371 espécies, representando $40,8 \%$ das ocorrências neotropicais. No Rio Grande do Sul, os primeiros registros de Culicinae foram realizados por Peryassú (1908), citando duas espécies. Posteriormente, Lutz et al. (1918) adicionaram outra. Pinto (1932) acrescenta oito espécies. Netto (1940) relaciona mais dezesseis. Pinto et al. (1940), ao reproduzir parte do trabalho de Netto (1940), incluiu mais uma. Mais tarde, Ruas-
Neto et al. (1994) citaram mais duas espécies. Finalmente, em 1996, a Fundação Nacional de Saúde (FUNASA/RS) registrou outra.

Tendo em vista os poucos estudos sobre os Culicinae no Rio Grande do Sul, aliados a necessidade de melhor conhecer a distribuição geográfica das espécies, como ferramenta para entender a epidemiologia das doenças transmitidas por vetores (Hutchings et al. 2002), este trabalho apresenta relação de espécies encontradas até o presente momento. Paralelamente, foram feitas atualizações de nomenclatura, incluindo os principais sinônimos e registros de ocorrência, além de possibilitar a implantação de duas coleções entomológicas regionais de referência.

\section{MATERIAL E MÉTODOS}

As caracterizações da área de estudo (Figura 1), incluindo coordenadas geográficas dos locais de coleta, bem como, detalhes dos procedimentos estão descritos em Cardoso et al. (2004).

Quando necessário, as estruturas da genitália masculina foram dissecadas e montadas em lâminas de vidro para a identificação específica. Para tanto, foram adotadas as técnicas 
de Forattini (1962) e Consoli \& Lourenço-de-Oliveira (1994). As espécies foram identificadas no Laboratório de Entomologia da Pontifícia Universidade Católica do Rio Grande do Sul (PUCRS), com auxílio de chaves dicotômicas contidas em Lane \& Cerqueira (1942), Lane (1953), Correa \& Ramalho (1956), Forattini (1962; 1965a,b; 2002), Darsie-Jr (1985) sendo, posteriormente, confirmadas no Laboratório de Taxonomia do Núcleo de Pesquisas Taxonômicas e Sistemática em Entomologia Médica da Faculdade de Saúde Pública da Universidade de São Paulo (NUPTEM-FSP/USP).

Além das coletas e revisão da literatura, foram examinados exemplares provenientes do Rio Grande do Sul nas seguintes Instituições: Laboratório Central de Saúde Pública do Estado do Rio Grande do Sul - LACEN/RS (LCRS), Museu Anchieta de Ciências Naturais (MAPA), Museu de Zoologia da Universidade de São Paulo (MZSP) e Faculdade de Saúde Pública da Universidade de São Paulo (FSP/USP).

A lista taxonômica relaciona as tribos, gêneros e espécies, em ordem alfabética. Os mapas de distribuição geográfica foram confeccionados através do programa TabWin32. A nomenclatura foi atualizada pelo catálogo de Guimarães (1997).

\section{RESULTADOS E DISCUSSÃO}

Relacionam-se 57 espécies incluídas em 15 gêneros e 7 tribos, conforme segue:

\section{CULICINAE}

Aedeomyiini

Aedeomyia Theobald, 1901

Aedeomyia (Aedeomyia) squamipennis (Lynch Arribálzaga, 1878)

Aedeomyia squamipennis; Netto (1940); Pinto et al. (1940); Marcondes et al. (2003)

Aedomyia [sic] sp.; Ruas-Neto et al. (1994).

Mat. Examinado: Santo Antônio da Patrulha, 05.iv.2001 (J. da C. Cardoso), 1 fêmea (MCTP); idem, 05.iv.2001 (J. da C. Cardoso), 1 fêmea (LCRS).

Registros no RS: Derrubadas, Santa Maria, *Santo Antônio da Patrulha, Porto Alegre.

Comentários: O registro de Ruas-Neto et al. (1994) omitindo o nome específico, certamente pode ser considerado como sendo Ad. squamipennis, já que esta é a única espécie do gênero, presente na Região Neotropical.

\footnotetext{
Aedini

Aedes Meigen, 1818

Aedes (Stegomyia) aegypti (Linnaeus, 1762)

Stegomyia calopus; Peryassú (1908); Lutz et al. (1918); Ronna (1924)

Stegomyia aegypti; Primio (1935); Netto (1940); Pinto et al. (1940)

Aedes aegypti; Ruas-Neto \& Silveira (1989).

Mat. Examinado: Crissiumal, 1997 (J. P. M. da Rocha), 1 fêmea (LCRS); Porto Alegre, 22.i.2002 (sem coletor) 1 fêmea (LCRS); idem, 05.ii.2002 (M. A. de Lima), 1 fêmea (LCRS); idem, 15.iv.2002 (R. F. Marques), 1 fêmea (LCRS).

Registros no RS: Crissiumal, Itaqui, Uruguaiana, Santa Maria, São Sebastião do Caí, Porto Alegre,
}

Quaraí, Santana do Livramento, São José do Norte, Rio Grande.

Comentários: Conforme informações da Divisão de Vigilância Ambiental em Saúde (DVAS), órgão da Secretaria Estadual de Saúde do RS, esta espécie já foi registrada em, aproximadamente, 350 municípios do Estado (Anexo I). Sua distribuição (Figura 2) pode ser verificada somando os registros bibliográficos e de material examinado do item registros no RS com o Anexo I, confeccionado em ordem alfabética de municípios.

Aedes (Stegomyia) albopictus (Skuse, 1894)

Mat. Examinado: Iraí, 28.iii.2002 (J. A. Teston), 20 fêmeas (MCTP); idem, 28.iii.2002 (J. A. Teston), 1 fêmea (LCRS); Caxias do Sul, 19.ii.2002 (P. Leite), 4 fêmeas (LCRS); Dois Irmãos, 04.iii.2002 (S. Heissler), 1 fêmea (LCRS); Santo Antônio da Patrulha, 29.iii.2002 (J. da C. Cardoso); 10 fêmeas (LCRS); Novo Hamburgo, 10.i.2002 (sem coletor), 2 fêmeas (LCRS), idem, 04.ii.2002 (sem coletor), 1 fêmea (LCRS); idem, 08.ii.2002 (S. L.?), 5 fêmeas (LCRS); Gravataí, 18.iii.2002 (A. Aguiar), 1 fêmea (LCRS); idem, 20.iii.2002 (T. de Oliveira), 1 fêmea (LCRS); Canoas, 04.iii.2002 (G. David-Silva), 4 fêmeas (LCRS); Porto Alegre, 20.iii.2003 (F. Paz), 1 macho (LCRS).

Registros no RS: Iraí, Caxias do Sul, Dois Irmãos, Santo Antônio da Patrulha, Novo Hamburgo, Gravataí, Canoas, Porto Alegre.

Comentários: Esta também é espécie de ampla distribuição (Figura 2) no Estado, segundo a DVAS, sendo, portanto, relacionada juntamente com $A e$. aegypti, no Anexo I.

\section{Haemagogus Williston, 1896}

Haemagogus (Conopostegus) leucocelaenus (Dyar \& Shannon, 1924)

Haemagogus (Stegoconops) leucomelas; Pinto (1932); Primio (1935)

Haemagogus leucomelas; Primio (1937); Netto (1940); Pinto et al. (1940)

Haemagogus (Con.) leucocelaenus; Vasconcelos et al. (2003).

Mat. Examinado: Derrubadas (Tenente Portela), i.1989 (A L. Ruas-Neto), 1 fêmea (LCRS); Santo Antônio das Missões, 28.ix.2001 (J. da C. Cardoso), 2 fêmeas, 2 machos (LCRS); Ernestina, 23.iii.2002 (A. P. R. Nunes), 4 fêmeas (MCTP).

Registros no RS: *Derrubadas, Garruchos, *Santo Antônio das Missões, *Ernestina, Gramado (Figura 2).

Comentários: Originalmente, Pinto (1932) registrou a ocorrência desta espécie no município de Taquara, na localidade que atualmente corresponde à cidade de Gramado.

Ochlerotatus Lynch Arribálzaga, 1891

Ochlerotatus (Ochlerotatus) albifasciatus (Macquart, 1838)

Culex albifasciatus; Lutz et al. (1918)

Culex albofaxiatus $[$ sic $]$; Ronna (1924)

Aedes (Ochlerotatus) albifasciatus; Primio (1935); Netto (1940); Pinto et al. (1940)

Mat. Examinado: Tramandaí, 10.v.1989 (A. L. Ruas-Neto), 5 fêmeas (LCRS); idem, 18.v.1989 (A. L. Ruas-Neto), 2 
fêmeas (LCRS); Quaraí, 14.vi.2002 (J. da C. Cardoso), 2 fêmeas (MCTP); Mostardas, 01.iii.2003 (S. B. Scherer) 32 fêmeas (LCRS); Tavares, 03.iii.2003 (F. de Mello) 18 fêmeas (LCRS).

Registros no RS: *Tramandaí, *Quaraí, *Mostardas, *Tavares, São José do Norte, Rio Grande.

Comentários: Oc. albifasciatus apresentou densidade muito elevada durante coleta realizada no Parque Nacional da Lagoa do Peixe no ano de 2003. Por este Parque receber, anualmente, aves que migram de áreas do hemisfério norte acometidas pelo Vírus do Nilo Ocidental, é importante continuar monitorando esta espécie no que diz respeito à sua possível associação na transmissão deste vírus aqui no Brasil.

Ochlerotatus (Ochlerotatus) crinifer (Theobald, 1903)

Aedes (Ochlerotatus) crinifer; Netto (1940); Pinto et al. (1940)

Aedes crinifer; Ruas-Neto et al. (1994)

Mat. Examinado: Derrubadas (Tenente Portela), i.1989 (A. L. Ruas-Neto), 13 fêmeas (LCRS); Santo Antônio das Missões, 28.ix.2001 (J. da C. Cardoso), 1 fêmea (MCTP); idem, 22.viii.2002 (I. A. da Costa), 5 fêmeas (LCRS); Caxias do Sul, 08.viii.2002 (J. A. S. Suñé), 2 fêmeas (MCTP); São Francisco de Paula, 20.x.2001 (J. A. S. Suñé), 2 fêmeas (MCTP); Canela, 21.iv.1989 (A. L. Ruas-Neto), 1 fêmea (LCRS); Santo Antônio da Patrulha, 05.iv.2001 (J. da C. Cardoso), 2 fêmeas (MCTP); Gravataí, 10.iv.2002 (R. C. Franco), 1 fêmea (LCRS); Viamão, 28.xi.2001 (J. da C. Cardoso), 1 fêmea (MCTP); Porto Alegre, 10.iv.1989 (A L. Ruas-Neto), 2 fêmeas (LCRS); idem, 16.iii.2002 (J. da C. Cardoso), 1 fêmea (FSP/USP); Quaraí, 14.vi.2002 (J. da C. Cardoso), 4 fêmeas (LCRS); idem, 15.vi.2002 (J. A. S. Suñé), 1 fêmea (MCTP); Camaquã, 05.viii.2002 (J. A. S. Suñé), 1 fêmea (LCRS).

Registros no RS: Derrubadas, *Santo Antônio das Missões, *Caxias do Sul, *São Francisco de Paula, *Canela, *Santo Antônio da Patrulha, *Gravataí, Porto Alegre, *Viamão, *Quaraí, *Camaquã.

\section{Ochlerotatus (Ochlerotatus) fluviatilis (Lutz, 1904)}

Danielsia tripunctata; Peryassú (1908)

Aedes (Culicelsa) fluviatilis; Pinto (1932); Primio (1935) Aedes (Finlaya) fluviatilis; Netto (1940); Pinto et al. (1940) Mat. Examinado: Derrubadas (Tenente Portela), i.1989 (A. L. Ruas-Neto), 3 fêmeas (LCRS); idem, 17.i.2002 (J. A. S. Suñé), 4 fêmeas (LCRS); idem, 17.i.2002 (J. da C. Cardoso), 4 fêmeas, 1 macho (MCTP); Ernestina, 22.iii.2002 (J. A. S. Suñé), 1 fêmea (MCTP); Caxias do Sul, 31.i.2002 (sem coletor), 1 fêmea (LCRS); Novo Hamburgo, 24.iv.2002 (S. ?), 1 fêmea (LCRS); Santa Cruz do Sul, 23.xi.1987 (S. M. Silveira), 1 macho (LCRS); idem, 08.iv.2002 (F. G. Pereira), 1 fêmea (MCTP); Canoas, 09.iv.2002 (A. Menezes), 1 fêmea (LCRS); Viamão, 04.iii.2002 (E. S. Santos), 1 fêmea (MCTP); Porto Alegre, 13.vi.2001 (L. S. Canali), 3 fêmeas (MCTP).

Registros no RS: *Derrubadas, *Ernestina, *Caxias do Sul, Gramado, *Santa Cruz do Sul, Santa Maria, *Novo Hamburgo, *Canoas, Porto Alegre, *Viamão. Comentários: Originalmente, Pinto (1932) refere o registro desta espécie para o município de Taquara, na localidade que atualmente corresponde à cidade de Gramado.
Ochlerotatus (Ochlerotatus) scapularis (Rondani, 1848)

Aedes (Ochlerotatus) scapularis; Pinto (1932); Primio (1935;1937); Netto (1940); Pinto et al. (1940); Vasconcelos et al. (2003)

Mat. Examinado: Sapiranga, 17.viii.1988 (A. L. Ruas-Neto), 1 fêmea (LCRS); Osório, 12.v.1989 (A. L. Ruas-Neto), 1 fêmea (LCRS); Canoas, 14.iii.2002 (G. David-Silva), 1 fêmea (LCRS); Gravataí, 10.iv.2002 (G. C. Souza), 1 fêmea (LCRS); Porto Alegre, 23.i.1988 (A. L. Ruas-Neto), 2 fêmeas (LCRS).

Registros no RS: Garruchos, Santo Antônio das Missões, Gramado, *Sapiranga, *Osório, *Gravataí, *Canoas, Porto Alegre.

Comentários: Originalmente, Pinto (1932) refere o registro desta espécie para o município de Taquara, na localidade que atualmente corresponde à cidade de Gramado.

Ochlerotatus (Ochlerotatus) serratus (Theobald, 1901)

Aedes (Ochlerotatus) serratus; Pinto (1932); Primio (1935); Netto (1940); Pinto et al. (1940); Vasconcelos et al. (2003) Mat. Examinado: Derrubadas (Tenente Portela), i.1989 (A L. Ruas-Neto), 3 fêmeas (LCRS); Santo Antônio das Missões, 28.ix.2001 (J. da C. Cardoso), 1 fêmea (MCTP). Registros no RS: *Derrubadas, Garruchos, *Santo Antônio das Missões, São Francisco de Paula, Venâncio Aires, Porto Alegre, Guaíba.

Ochlerotatus (Protomacleaya) terrens (Walker, 1956)

Mat. Examinado: Derrubadas (Tenente Portela), i.1989 (A.

L. Ruas-Neto), 1 fêmea (MCTP).

Registro no RS: Derrubadas.

Comentários: Nova ocorrência para o Estado.

Psorophora Robineau-Desvoidy, 1827

Psorophora (Grabhamia) confinnis (Lynch Arribálzaga, 1891)

Psorophora (Grabhamia) confinis [sic]; Netto (1940); Pinto et al. (1940)

Mat. Examinado: Candelária, 12.iii.2002 (A. Silva), 1 fêmea (MCTP); Santo Antônio da Patrulha, 05.iv.2001 (J. da C. Cardoso), 2 fêmeas (MCTP); idem, 08.xii.2001 (J. da C. Cardoso), 4 fêmeas (LCRS); Canoas, 12.iii.2002 (J. da C. Cardoso), 1 fêmea (MCTP) .

Registros no RS: *Candelária, *Santo Antônio da Patrulha, *Canoas, Porto Alegre.

Psorophora (Grabhamia) varinervis Edwards, 1922

Psorophora (Grabhamia) varinervis; Pinto (1932); Primio (1935; 1937); Netto (1940); Pinto et al. (1940); Lane (1953)

Registros no RS: Gramado, Viamão, Porto Alegre.

Comentários: Originalmente, Pinto (1932) refere o primeiro registro da espécie no Brasil, para o município de Taquara, na localidade que atualmente corresponde à cidade de Gramado. Desde então, o RS é o único estado com informação de ocorrência desta espécie, embora não se verifique a disponibilidade de exemplares em coleções, fato que gera dúvida e indica que novas coletas devam ser 
realizadas para que este registro seja confirmado.

Psorophora (Janthinosoma) albigenu (Peryassú, 1908)

Psorophora (Janthinosoma) varipes; Netto (1940); Pinto et al. (1940)

Registros no RS: Venâncio Aires, Porto Alegre.

Comentários: Segundo Guimarães (1997), Ps. varipes é uma espécie de identidade e distribuição incertas, sendo as populações da América Central, provavelmente, diferentes das norte americanas. Os exemplares identificados no continente sul americano correspondem a Ps. albigenu.

\section{Psorophora (Janthinosoma) discrucians (Walker, 1856)}

Mat. Examinado: Porto Alegre, $10 . i v .1989$ (A. L. RuasNeto), 8 fêmeas (LCRS); idem, 11.iv.1989 (A. L. RuasNeto), 1 macho (LCRS); Camaquã, 24.ii.1990 (A. L. RuasNeto), 1 fêmea (LCRS).

Registros no RS: Porto Alegre, Camaquã.

Comentários: Nova ocorrência para o Estado.

Psorophora (Janthinosoma) ferox (von Humboldt, 1819)

Psorophora (Janthinosoma) ferox; Pinto (1932); Primio (1935; 1937); Netto (1940); Pinto et al. (1940)

Psorophora (Jan.) feroz [sic]; Vasconcelos et al. (2003)

Mat. Examinado: Derrubadas (Tenente Portela), i.1989 (A. L. Ruas-Neto), 16 fêmeas (LCRS); Santo Antônio das Missões, 26.ix.2001 (J. da C. Cardoso), 5 fêmeas (MCTP); idem, 28.ix.2001 (J. da C. Cardoso), 6 fêmeas (LCRS); Ernestina, 23.iii.2002 (L. de F. Garrido), 2 fêmeas (MCTP); São Francisco de Paula, 20.x.2001 (J. A. S. Suñé), 1 fêmea (MCTP); Viamão, 28.xi.2001 (J. da C. Cardoso), 1 fêmea (MCTP).

Registros no RS: *Derrubadas, Garruchos, *Santo Antônio das Missões, *São Francisco de Paula, Gramado, Venâncio Aires, Porto Alegre, *Viamão.

Comentários: Originalmente, Pinto (1932) refere o registro desta espécie para o município de Taquara, na localidade que atualmente corresponde à cidade de Gramado.

\section{Psorophora (Psorophora) ciliata (Fabricius, 1794)}

Psorophora (Psorophora) ciliata; Netto (1940); Pinto et al. (1940)

Registro no RS: Porto Alegre.

\section{Psorophora (Psorophora) saeva Dyar \& Knab, 1906}

Mat. Examinado: Derrubadas (Tenente Portela), i.1989 (A. L. Ruas-Neto), 1 macho, 1 genitália masculina (LCRS). Registro no RS: Derrubadas.

Comentários: Nova ocorrência para o Estado. Eventualmente, Psorophora saeva pode ser confundida com Ps. lineata. No entanto, Ps. saeva é espécie do norte e leste da América do Sul, ocorrendo desde a Venezuela até a Argentina, passando pelo Brasil; enquanto Ps. lineata se estende pelo oeste da América do Sul, da Bolívia ao México, América Central e do Norte e Suriname.
Ambas ocorrem na Venezuela, Trinidad e Suriname (Stone 1967).

\section{Culicini}

Culex Linnaeus, 1758

Culex (Culex) bidens Dyar, 1922

Mat. Examinado: Cidreira, 21.iv.2002 (J. A. S. Suñé), 1 macho (MCTP); Porto Alegre, 03.v.2002 (L. de F. Garrido), 1 macho (MCTP); Quaraí, 15.vi.2002 (J. A. S. Suñé), 1 macho, 1 genitália masculina (LCRS).

Registros no RS: Porto Alegre, Quaraí, Cidreira.

Comentários: Nova ocorrência para o Estado.

\section{Culex (Culex) chidesteri Dyar, 1921}

Culex (Culex) chidesteri; Netto (1940); Pinto et al. (1940) Mat. Examinado: Osório, 20.iv.2002 (J. A. S. Suñé), 2 fêmeas (FSP/USP); Santo Antônio da Patrulha, 29.iii.2002 (J. da C. Cardoso), 1 macho (MCTP); Quaraí, 14.vi.2002 (J. da C. Cardoso), 3 fêmeas (MCTP); idem, 15.vi.2002 (J. A. S. Suñé), 1 fêmea, 1 macho (MCTP).

Registros no RS: Torres, *Santo Antônio da Patrulha, *Osório, *Quaraí.

Comentários: Foram confirmados os registros de Netto (1940) e Pinto et al. (1940).

\section{Culex (Culex) dolosus (Lynch Arribálzaga, 1891)}

Mat. Examinado: Santo Antônio da Patrulha, 29.iii.2002 (J. da C. Cardoso), 1 macho (LCRS); Viamão, 28.xi.2001 (J. A. S. Suñé), 1 fêmea (LCRS); Quaraí, 14.vi.2002 (J. da C. Cardoso), 2 fêmeas (MCTP); idem, 14.vi.2002 (J. da C. Cardoso), 2 fêmeas (FSP/USP).

Registros no RS: Santo Antônio da Patrulha, Viamão, Quaraí.

Comentários: Nova ocorrência para o Estado.

\section{Culex (Culex) quinquefasciatus Say, 1823}

Culex (Culex) quinquefasciatus; Pinto (1932); Primio (1935); Netto (1940); Pinto et al. (1940); Vianna et al. (1996a,b)

Culex quinquefasciatus; Ruas-Neto \& Silveira (1989); RuasNeto et al. (1994)

Mat. Examinado: Iraí, 11.viii.2001 (J. A. Teston), 1 fêmea (MCTP); idem, 12.viii.2001 (J. A. Teston), 2 fêmeas (MCTP); idem, 24.iii.2002 (J. A. Teston), 1 fêmea (MCTP); idem, 27.iii.2002 (J. A. Teston), 7 fêmeas (MCTP); idem, 15.x.2002 (J. A. Teston), 3 fêmeas (MCTP); Santo Antônio das Missões, 25.ix.2001 (J. da C. Cardoso), 1 fêmea (MCTP); Porto Alegre, 20.vi.2002 (J. A. S. Suñé), 1 fêmea (MCTP).

Registros no RS: *Iraí, Passo Fundo, *Santo Antônio das Missões, Soledade, São Francisco de Paula, Júlio de Castilhos, Torres, Venâncio Aires, Gramado, Feliz, Santa Maria, Taquari, Santo Antônio da Patrulha, Osório, Gravataí, Cachoeira do Sul, Porto Alegre, Guaíba, Pinheiro Machado, Pelotas, Herval, Rio Grande.

Comentários: Originalmente, Pinto (1932) refere o registro desta espécie para os municípios de Taquara, na localidade que hoje corresponde à cidade de Gramado, e Cacimbinhas, atualmente, Pinheiro Machado. 
Culex (Culex) saltanensis Dyar, 1928

Culex saltanensis; Ruas-Neto et al. (1994)

Registros no RS: Teutônia, Feliz.

\section{Culex (Culex) sp.}

Culex (Culex) coronator; Pinto (1932); Primio (1935); Netto (1940); Pinto et al. (1940)

Culex coronator; Ruas-Neto et al. (1994)

Culex (Cux.) coronator; Vasconcelos et al. (2003)

Mat. Examinado: Derrubadas (Tenente Portela), i.1989 (A.

L. Ruas-Neto), 4 fêmeas (LCRS); Santo Antônio das Missões, 28.ix.2001 (J. da C. Cardoso), 02 fêmeas (MCTP); idem, 28.ix.2001 (J. da C. Cardoso), 2 fêmeas (FSP/USP); Quaraí, 14.vi.2002 (J. da C. Cardoso), 4 fêmeas (MCTP).

Registros no RS: Derrubadas, Garruchos, *Santo Antônio das Missões, Soledade, São Francisco de Paula, Júlio de Castilhos, Feliz, Porto Alegre, *Quaraí.

Comentários: Os indivíduos identificados como Culex (Culex) sp. são morfologicamente semelhantes às espécies do grupo coronator. No entanto, como foram examinadas somente fêmeas, não foi possível uma identificação específica.

Culex (Melanoconion) ribeirensis Forattini \& Sallum, 1985

Mat. Examinado: Derrubadas, 17.i.2002 (J. da C. Cardoso), 1 fêmea, 1macho (MCTP); idem, 17.i.2002 (J. A. S. Suñé), 1 fêmea (MCTP); Santo Antônio das Missões, 28.ix.2001 (J. da C. Cardoso), 3 fêmeas (MCTP); idem, 22.viii.2002 (I. A. da Costa), 7 fêmeas (LCRS); Viamão, 28.xi.2001 (J. A. S. Suñé), 1 fêmea (FSP/USP); idem, 28.xi.2001 (J. da C. Cardoso), 1 fêmea (FSP/USP); Quaraí, 15.vi.2002 (J. A. S. Suñé), 3 fêmeas (MCTP)

Registros no RS: Derrubadas, Santo Antônio das Missões, São Francisco de Paula, Viamão, Quaraí.

Comentários: Nova ocorrência para o Estado.

Culex (Microculex) imitator Theobald, 1903

Culex (Microculex) imitator; Netto (1940); Pinto et al. (1940)

Registro no RS: São Francisco de Paula.

Culex (Phenacomyia) corniger Theobald, 1903

Culex (Cux.) corriger [sic]; Vasconcelos et al. (2003)

Registros no RS: Garruchos, Santo Antônio das Missões.

Lutzia (Lutzia) bigoti (Bellardi, 1862)

Lutzia bigoti; Netto (1940); Pinto et al. (1940); Ruas-Neto et al. (1994)

Registros no RS: Torres, Feliz, Porto Alegre.

\section{Mansoniini}

Coquillettidia Dyar, 1905

Coquillettidia (Rhynchotaenia) fasciolata (Lynch Arribálzaga, 1891)

Taeniorhynchus (Rhynchotaenia) fasciolatus; Netto (1940); Pinto et al. (1940)

Registro no RS: Porto Alegre.
Coquillettidia (Rhynchotaenia) nigricans (Coquillet, 1904)

Mat. Examinado: Canela, 22.xi.2002 (sem coletor), 1 fêmea (LCRS); Viamão, 28.xi.2001 (J. da C. Cardoso), 5 fêmeas (MCTP)

Registros no RS: Canela, Viamão.

Comentários: Nova ocorrência para o Estado.

Coquillettidia (Rhynchotaenia) shannoni (Lane \& Antunes, 1937)

Mat. Examinado: Viamão, 28.xi.2001 (J. da C. Cardoso), 1 fêmea (MCTP); idem, 28.xi.2001 (J. da C. Cardoso), 2 fêmeas (LCRS).

Registro no RS: Viamão.

Comentários: Nova ocorrência para o Estado.

Coquillettidia (Rhynchotaenia) venezuelensis (Theobald, 1912)

Mat. Examinado: Derrubadas, 17.i.2002 (J. da C. Cardoso), 5 fêmeas (LCRS); Santo Antônio da Patrulha, 05.iv.2001 (J. da C. Cardoso), 1 fêmea (FSP/USP); idem, 08.xii.2001 (J. da C. Cardoso), 1 fêmea (FSP/USP); Viamão, 28.xi.2001 (J. da C. Cardoso), 8 fêmeas (MCTP); idem, 28.xi.2001 (J. A. S. Suñé), 4 fêmeas (LCRS)

Registros no RS: Derrubadas, Santo Antônio da Patrulha, Viamão.

Comentários: Nova ocorrência para o Estado.

Mansonia Blanchard, 1901

Mansonia (Mansonia) humeralis Dyar \& Knab, 1916

Mat. Examinado: Santo Antônio da Patrulha, 05.iv.2001 (J. da C. Cardoso), 9 fêmeas (LCRS); idem, 08.xii.2001 (J. da C. Cardoso), 2 machos (LCRS); idem, 08.xii.2001 (J. da C. Cardoso), 2 fêmeas (FSP/USP); idem, 08.xii.2001 (J. da C. Cardoso), 3 fêmeas, 3 machos (MCTP).

Registro no RS: Santo Antônio da Patrulha.

Comentários: Nova ocorrência para o Estado.

Mansonia (Mansonia) pseudotitillans (Theobald, 1901)

Taeniorhynchus (Taeniorhynchus) pseudotitillans; Netto, 1940; Pinto et al., 1940

Registro no RS: Porto Alegre.

\section{Mansonia (Mansonia) sp.}

Mat. Examinado: Iraí, 24.iii.2002 (J. A. Teston) 1 fêmea (LCRS); Santo Antônio da Patrulha, 05.iv.2001 (J. da C. Cardoso), 2 fêmeas (LCRS).

Registros no RS: Iraí, Santo Antônio da Patrulha. Comentários: Espécie próxima a Ma. indubitans.

Mansonia (Mansonia) titillans (Walker, 1848)

Taeniorhynchus (Taeniorhynchus) titillans; Netto (1940); Pinto et al. (1940); Ruas-Neto \& Silveira (1989) Mansonia (Man.) titillans; Vasconcelos et al. (2003)

Mat. Examinado: Derrubadas, 17.i.2002 (J. A. S. Suñé), 1 fêmea (MCTP); Ernestina, 22.iii.2002 (J. A. S. Suñé), 2 fêmeas (MCTP); Teutônia, 06.ii.1990 (E. R. C. Colares), 1 fêmea (MCTP); Osório, 20.iv.2002 (J. A. S. Suñé), 1 fêmea (MCTP); Santo Antônio da Patrulha, 05.iv.2001 (J. da C. Cardoso), 16 fêmeas (LCRS); idem, 05.iv.2001 (J. da 
C. Cardoso), 5 fêmeas (FSP/USP); Cidreira, 21.iv.2002 (J. A. S. Suñé), 1 fêmea (MCTP); Viamão, 28.xi.2001 (J. da C. Cardoso), 5 fêmeas (MCTP); Porto Alegre, 16.iii.2002 (J. da C. Cardoso), 1 fêmea (MCTP); idem, 15.iv.2002 (C. Algayer), 1 fêmea (MCTP).

Registros no RS: *Derrubadas, *Ernestina, Santo Antônio das Missões, *Teutônia, *Santo Antônio da Patrulha, *Osório, Porto Alegre, *Viamão, *Cidreira.

Mansonia (Mansonia) wilsoni (Barreto \& Coutinho, 1944)

Mat. Examinado: Porto Alegre, iii.1945 (E. Ronna) 2 exemplares (MZSP)

Registros no RS: Porto Alegre.

Comentários: Nova ocorrência para o Estado. Os espécimes estão depositados na coleção entomológica do MZSP.

\section{Sabethini}

Limatus Theobald, 1901

Limatus durhami Theobald, 1901

Mat. Examinado: Nova Bassano, 17.iii.2003 (J. A. Rossolini), 2 larvas (LCRS); Nova Petrópolis, 17.iii.2003 (E. Volta), 2 larvas (LCRS)

Registro no RS: Nova Bassano, Nova Petrópolis. Comentários: Nova ocorrência para o Estado. Não era objetivo do trabalho capturar formas imaturas, no entanto, este material foi utilizado devido à inexistência de exemplares adultos.

Limatus flavisetosus Oliveira Castro, 1935

Mat. Examinado: Porto Alegre, 20.iii.2001 (V. Baldasso), 1 larva (LCRS)

Registro no RS: Porto Alegre

Comentários: Nova ocorrência para o Estado. Valem as mesmas considerações feitas para Li. durhami.

Sabethes Robineau-Desvoidy, 1827

Sabethes (Peytonulus) aurescens (Lutz, 1905)

Sabethes aurescens; Marcondes et al. (2003)

Mat. Examinado: Derrubadas (Tenente Portela), i.1989 (A. L. Ruas-Neto), 4 fêmeas (MCTP); idem, i.1989 (A. L. Ruas-Neto), 4 fêmeas (LCRS); São Francisco de Paula, 20.x.2001 (J. A. S. Suñé), 3 fêmeas (FSP/USP).

Registro no RS: *Derrubadas, São Francisco de Paula.

Sabethes (Peytonulus) soperi Lane \& Cerqueira, 1942

Mat. Examinado: Santo Antônio das Missões, 25.ix.2001 (J. da C. Cardoso), 1 fêmea (LCRS).

Registro no RS: Santo Antônio das Missões.

Comentários: Nova ocorrência para o Estado.

Sabethes (Sabethes) albiprivus Theobald, 1903

Sabethes (Sab.) albiprivus; VASCONCELOS et al. (2003) Mat. Examinado: Derrubadas (Tenente Portela), i.1989 (A. L. Ruas-Neto), 1 fêmea (MCTP); idem, i.1989 (A. L. RuasNeto), 1 fêmea (LCRS).

Registro no RS: Derrubadas, Garruchos, Santo Antônio das Missões.
Sabethes (Sabethes) purpureus (Theobald, 1907)

Mat. Examinado: Derrubadas (Tenente Portela), i.1989 (A. L. Ruas-Neto), 1 fêmea (LCRS).

Registro no RS: Derrubadas.

Comentários: Nova ocorrência para o Estado.

Sabethes (Sabethinus) melanonymphe Dyar, 1924

Sabethoides albiprivatus; PINTO et al. (1940)

Registro no RS: São Francisco de Paula.

Sabethes (Sabethinus) xyphydes Harbach, 1994

Mat. Examinado: Derrubadas (Tenente Portela), i.1989 (A. L. Ruas-Neto), 1 fêmea (LCRS).

Registro no RS: Derrubadas.

Comentários: Nova ocorrência para o Estado.

Trichoprosopon Theobald, 1901

Trichoprosopon compressum Lutz, 1905

Mat. Examinado: Derrubadas (Tenente Portela), i.1989 (A. L. Ruas-Neto), 1 fêmea (LCRS).

Registro no RS: Derrubadas.

Comentários: Nova ocorrência para o Estado.

Trichoprosopon pallidiventer (Lutz, 1905)

Goeldia pallidiventer; Pinto (1932); Primio (1935; 1937); Netto (1940); Pinto et al. (1940).

Mat. Examinado: Derrubadas (Tenente Portela), i.1989 (A. L. Ruas-Neto), 1 fêmea (LCRS); São Francisco de Paula, 19.x.2001 (J. A. S. Suñé), 16 fêmeas (MCTP); idem, 19.x.2001 (J. da C. Cardoso), 1 macho (FSP/USP); idem, 20.x.2001 (J. da C. Cardoso), 1 fêmea (FSP/USP); idem, 20.x.2001 (J. A. S. Suñé), 4 fêmeas (LCRS).

Registros no RS: *Derrubadas, *São Francisco de Paula, Gramado

Comentários: Originalmente, Pinto (1932) refere o registro desta espécie para o município de Taquara, na localidade que atualmente corresponde à cidade de Gramado.

Wyeomyia Theobald, 1901

Wyeomyia (Phoniomyia) davisi (Lane \& Cerqueira, 1942)

Mat. Examinado: Viamão, 16.xi.2001 (J. da C. Cardoso), 3 fêmeas (LCRS); idem, 29.xi.2001 (J. da C. Cardoso), 2 fêmeas (MCTP); idem, 29.xi.2001 (J. A. S. Suñé), 1 fêmea (MCTP).

Registro no RS: Viamão.

Comentários: Nova ocorrência para o Estado.

Wyeomyia (Phoniomyia) lopesi (Corrêa \& Ramalho, 1956)

Mat. Examinado: Viamão, 16.xi.2001 (J. da C. Cardoso), 1 fêmea (LCRS).

Registro no RS: Viamão.

Comentários: Nova ocorrência para o Estado.

Wyeomyia (Phoniomyia) quasilongirostris (Theobald, 1907)

Mat. Examinado: Viamão, 16.xi.2001 (J. da C. Cardoso), 2 
fêmeas (LCRS); idem, 16.xi.2001 (J. da C. Cardoso), 1 fêmea (FSP/USP).

Registro no RS: Viamão.

Comentários: Nova ocorrência para o Estado.

Wyeomyia (Wyeomyia) codiocampa Dyar \& Knab, 1907

Wyeomyia codiocampa; Netto (1940); Pinto et al. (1940); Lane (1953)

Wyeomyia (Wyeomyia) codiocampa; Lane \& Cerqueira (1942)

Registro no RS: São Francisco de Paula.

Wyeomyia (Wyeomyia) limai Lane \& Cerqueira, 1942

Mat. Examinado: Derrubadas (Tenente Portela), i.1989 (A. L. Ruas-Neto), 1 fêmea (MCTP); Santo Antônio das Missões, 28.ix.2001 (J. da C. Cardoso), 2 fêmeas (LCRS); São Francisco de Paula, 20.x.2001 (J. A. S. Suñé), 2 fêmeas (MCTP)

Registros no RS: Derrubadas, Santo Antônio das Missões, São Francisco de Paula.

Comentários: Nova ocorrência para o Estado.

Wyeomyia serratoria (Dyar \& Nuñez Tovar, 1927)

Mat. Examinado: Derrubadas (Tenente Portela), i.1989 (A. L. Ruas-Neto), 1 fêmea (LCRS).

Registro no RS: Derrubadas.

Comentários: Nova ocorrência para o Estado. Guimarães (1997) considerou Wy. serratoria, juntamente com outras 47 espécies, como sendo de subgênero incerto.

\section{Toxorhynchitini}

Toxorhynchites Theobald, 1901

Toxorhynchites (Lynchiella) sp.

Megarhynus (Megarhynus) sp.; Netto (1940); Pinto et al. (1940)

Mat. Examinado: Sapucaia do Sul, 08.x.1984 (C. J. Becker), 1 fêmea (MCNZ 69086); Parecy Novo, 24.viii.1932 (sem coletor), 1 fêmea (MAPA A27 G1); Lindolfo Collor, 21.xii.2002 (K. Bauermann), 1 fêmea (LCRS).

Registros no RS: *Lindolfo Collor, *Pareci Novo, * Sapucaia do Sul, Porto Alegre.

\section{Uranotaeniini}

Uranotaenia Lynch Arribálzaga, 1891

Uranotaenia (Uranotaenia) calosomata Dyar \& Knab, 1907

Uranotaenia (Ura.) calosomata; Vasconcelos et al. (2003) Registro no RS: Garruchos, Santo Antônio das Missões.

Uranotaenia (Uranotaenia) geometrica Theobald, 1901

Uranotaenia geometrica; Netto (1940); Pinto et al. (1940) Registro no RS: Porto Alegre.

Uranotaenia (Uranotaenia) lowii Theobald, 1901 Uranotaenia lowii; Netto (1940); Pinto et al. (1940)
Uranotaenia (Ura.) lowii; Vasconcelos et al. (2003)

Mat. Examinado: Osório, 20.iv.2002 (J. A. S. Suñé), 1 fêmea (MCTP); Tramandaí, 18.v.1989 (A. L. Ruas-Neto), 1 fêmea (LCRS); Santo Antônio da Patrulha, 08.xii.2001 (J. da C. Cardoso), 1 macho (MCTP); Porto Alegre, 11.iv.1989 (A. L. Ruas-Neto), 1 fêmea (LCRS); idem, 08.v.2002 (J. da C. Cardoso), 1 fêmea (MCTP).

Registros no RS: Garruchos, Santo Antônio das Missões, *Santo Antônio da Patrulha, *Osório, *Tramandaí, Porto Alegre.

Uranotaenia (Uranotaenia) nataliae Lynch Arribálzaga, 1891

Uranotaenia nataliae; Netto (1940); Pinto et al. (1940) Registro no RS: Porto Alegre.

Nesta relação de espécies, constam representantes dos gêneros Aedeomyia (1), Aedes (2), Coquillettidia (4), Culex (9), Haemagogus (1), Limatus (2), Lutzia (1), Mansonia (5), Ochlerotatus (6), Psorophora (7), Sabethes (6), Toxorhynchites (1), Trichoprosopon (2), Uranotaenia (4) e Wyeomyia (6) que estão incluídos na tabela 1. A distribuição é dada de acordo com as zonas fisiográficas do Estado.

As espécies Coquillettidia nigricans, Coquillettidia shannoni, Coquillettidia venezuelensis, Culex bidens, Culex

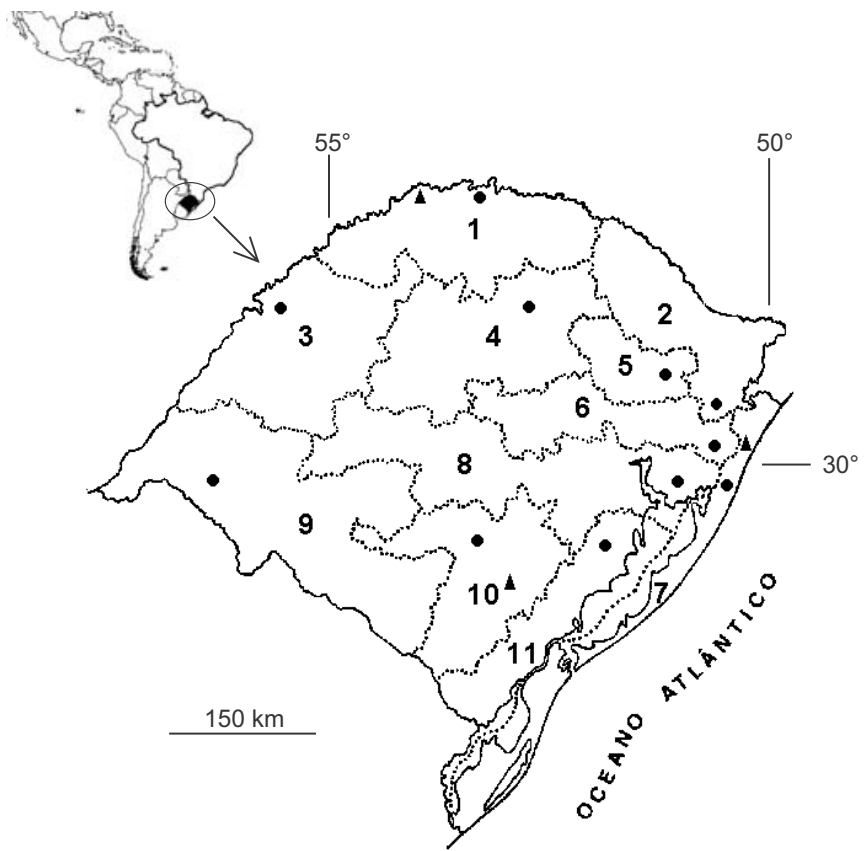

Fig. 1. Zonas fisiográficas do Rio Grande do Sul (segundo AREND, 1997), com os locais onde foram realizadas as coletas. 1 - ALTO URUGUAI: $\Delta$ Iraí, - Derrubadas; 2 - CAMPOS DE CIMA DA SERRA: São Francisco de Paula; 3 - MISSÕES: Santo Antônio das Missões; 4 - PLANALTO MÉDIO: Ernestina; 5 - ENCOSTA SUPERIOR DO NORDESTE: Caxias do Sul; 6 - ENCOSTA INFERIOR DO NORDESTE: Santo Antônio da Patrulha; 7 - LITORAL: - Osório, A Cidreira; 8 - DEPRESSÃO CENTRAL: Viamão; 9 - CAMPANHA: Quaraí; 10 - SERRA DO SUDESTE: A Caçapava do Sul, • Piratini; 11 - ENCOSTA DO SUDESTE: Camaquã. 

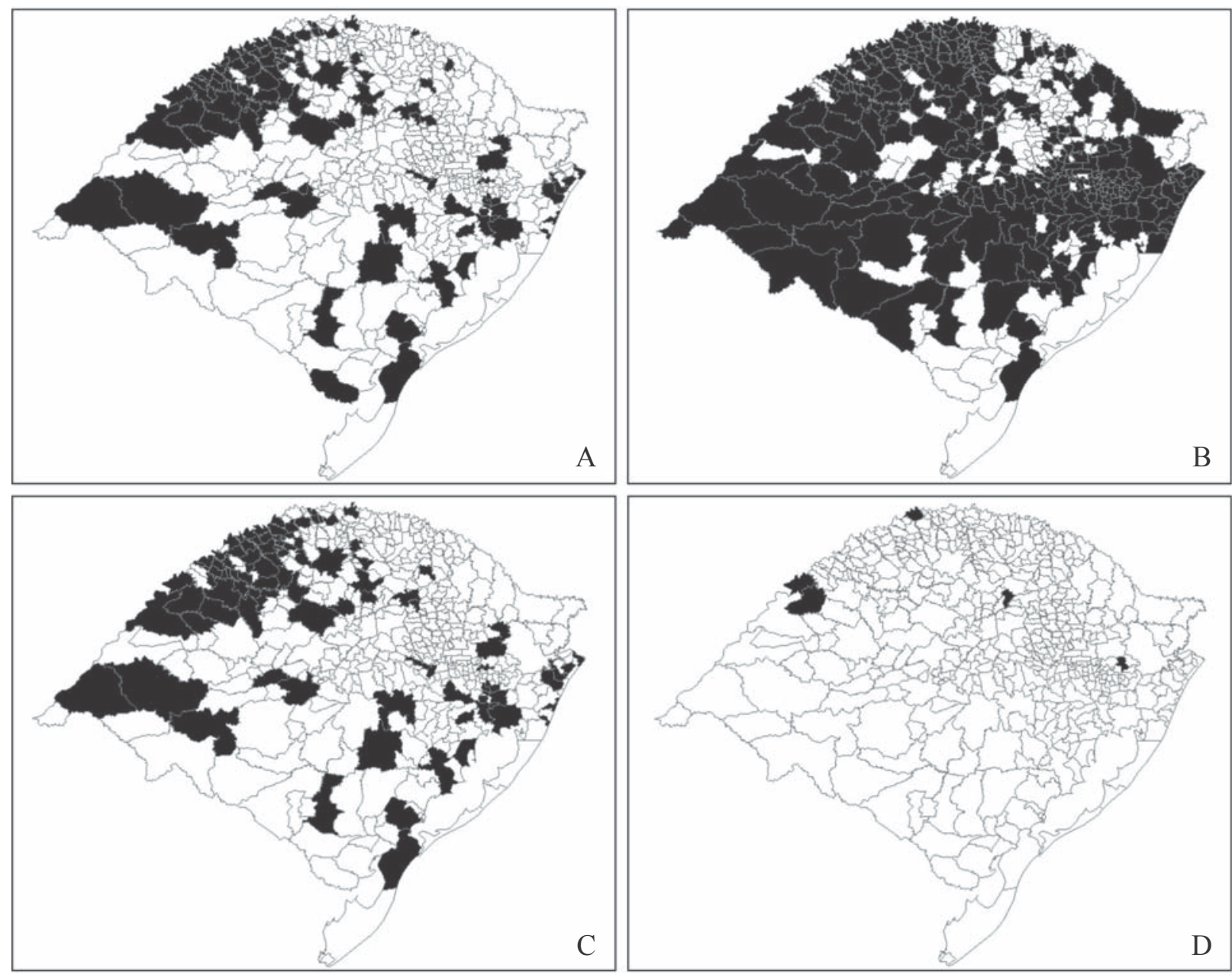

Fig. 2. Distribuição dos principais culicíneos de relevância sanitária presentes no RS. A) Aedes aegypti; B) Aedes albopictus; C) Aedes aegypti e Aedes albopictus ocorrendo simultaneamente; D) Haemagogus leucocelaenus.

dolosus, Culex ribeirensis, Limatus durhami, Limatus flavisetosus, Mansonia humeralis, Mansonia wilsoni, Ochlerotatus terrens, Psorophora discrucians, Psorophora saeva, Sabethes purpureus, Sabethes soperi, Sabethes xyphydes, Trichoprosopon compressum, Wyeomyia davisi, Wyeomyia limai, Wyeomyia lopesi, Wyeomyia quasilongirostris e Wyeomyia serratoria, representam novos registros para o Rio Grande do Sul.

Foram ampliadas as ocorrências de Aedeomyia squamipennis, Culex chidesteri, Culex quinquefasciatus, Haemagogus leucocelaenus, Mansonia titillans, Ochlerotatus albifasciatus, Ochlerotatus crinifer, Ochlerotatus fluviatilis, Ochlerotatus scapularis, Ochlerotatus serratus, Psorophora confinnis, Psorophora ferox, Sabethes aurescens, Trichoprosopon pallidiventer e Uranotaenia lowii.

Os exemplares capturados no Rio Grande do Sul, resultantes dos trabalhos de Ruas-Neto \& Silveira (1989) e Ruas-Neto et al. (1994), encontravam-se no LACEN/RS, sem fazer parte de coleção científica. Esses exemplares, juntamente com alguns, agregados recentemente, e outros, coletados para este estudo, foram devidamente tombados, originando uma coleção de referência da fauna do Estado. $\mathrm{O}$ acervo incorporado à coleção entomológica do MCTP constitui outra importante coleção de referência regional.

A realização de capturas nas zonas fisiográficas forneceu considerável amostra da fauna de culicíneos dos grandes ecossistemas do Estado, resguardadas as características de espécies silvestres, periurbanas e urbanas. Desta forma, pôdese verificar que Ae. aegypti e Ae. albopictus (Figura 2), Cx. quinquefasciatus e Oc. crinifer são amplamente distribuídas no Rio Grande do Sul. O registro de Ae. aegypti e Ae. albopictus em todas as zonas fisiográficas deve-se ao fato de serem espécies alvo do Programa de Controle da Febre Amarela e Dengue (PCFAD), que acumula diversas informações sobre as mesmas desde 1995. A presença da última, no Estado, foi verificada em 1996 pela FUNASA/RS, mas esta informação ficou restrita ao Sistema de Informação de Febre Amarela e Dengue (SISFAD) do PCFAD e aos profissionais de saúde que atuam no programa de controle. A pouca abrangência 
Tabela I. Registros de culicíneos no Rio Grande do Sul, por zonas fisiográficas: AUr (Alto Uruguai), Cam (Campanha), CCS (Campos de Cima da Serra), DeC (Depressão Central), ESu (Encosta do Sudeste), EIN (Encosta Inferior do Nordeste), Lit (Litoral), Mis (Missões), PIM (Planalto Médio), SSu (Serra do Sudeste).

\begin{tabular}{|c|c|c|c|c|c|c|c|c|c|c|c|}
\hline TÁXON & AUr & Cam & $\mathrm{CCS}$ & $\mathrm{DeC}$ & $\mathrm{ESu}$ & EIN & ESN & Lit & Mis & P1M & $\mathrm{SSu}$ \\
\hline 1. Aedeomyia squamipennis & $\bullet$ & & & $\bullet$ & & $\bullet$ & & & & & \\
\hline 2. Aedes aegypti & • & $\bullet$ & $\bullet$ & $\bullet$ & $\bullet$ & $\bullet$ & $\bullet$ & $\bullet$ & $\bullet$ & $\bullet$ & • \\
\hline 3. Aedes albopictus & $\bullet$ & $\bullet$ & $\bullet$ & $\bullet$ & $\bullet$ & $\bullet$ & $\bullet$ & $\bullet$ & $\bullet$ & $\bullet$ & $\bullet$ \\
\hline 4. Coquillettidia fasciolata & & & & $\bullet$ & & & & & & & \\
\hline 5. Coquillettidia nigricans & & & & $\bullet$ & & $\bullet$ & & & & & \\
\hline 6. Coquillettidia shannoni & & & & $\bullet$ & & & & & & & \\
\hline 7. Coquillettidia venezuelensis & $\bullet$ & & & $\bullet$ & & $\bullet$ & & & & & \\
\hline 8. Culex corniger & • & & & & & & & & & & \\
\hline 9. Culex bidens & & $\bullet$ & & $\bullet$ & & & & $\bullet$ & & & \\
\hline 10. Culex chidesteri & & $\bullet$ & & & & $\bullet$ & & $\bullet$ & & & \\
\hline 11. Culex dolosus & & $\bullet$ & & $\bullet$ & & $\bullet$ & & & & & \\
\hline 12. Culex imitator & & & • & & & & & & & & \\
\hline 13. Culex quinquefasciatus & $\bullet$ & & - & $\bullet$ & $\bullet$ & $\bullet$ & & $\bullet$ & $\bullet$ & $\bullet$ & $\bullet$ \\
\hline 14. Culex ribeirensis & $\bullet$ & $\bullet$ & $\bullet$ & $\bullet$ & & & & & $\bullet$ & & \\
\hline 15. Culex saltanensis & & & & & & $\bullet$ & & & & & \\
\hline 16. Culex (Culex) sp. & $\bullet$ & • & $\bullet$ & • & & $\bullet$ & & & $\bullet$ & $\bullet$ & \\
\hline 17. Haemagogus leucocelaenus & $\bullet$ & & & & & $\bullet$ & & & $\bullet$ & $\bullet$ & \\
\hline 18. Limatus durhami & & & & & & & $\bullet$ & & & & \\
\hline 19. Limatus flavisetosus & & & & $\bullet$ & & & & & & & \\
\hline 20. Lutzia bigoti & & & & $\bullet$ & & $\bullet$ & & $\bullet$ & & & \\
\hline 21. Mansonia humeralis & & & & & & $\bullet$ & & & & & \\
\hline 22. Mansonia pseudotitillans & & & & $\bullet$ & & & & & & & \\
\hline 23. Mansonia (Mansonia) sp. & $\bullet$ & & & & & $\bullet$ & & & & & \\
\hline 24. Mansonia titillans & $\bullet$ & & & $\bullet$ & & $\bullet$ & & $\bullet$ & & $\bullet$ & \\
\hline 25. Mansonia wilsoni & & & & $\bullet$ & & & & & & & \\
\hline 26. Ochlerotatus albifasciatus & & $\bullet$ & & & & & & $\bullet$ & & & \\
\hline 27. Ochlerotatus crinifer & $\bullet$ & $\bullet$ & $\bullet$ & $\bullet$ & $\bullet$ & $\bullet$ & $\bullet$ & & $\bullet$ & & \\
\hline 28. Ochlerotatus fluviatilis & $\bullet$ & & & $\bullet$ & & $\bullet$ & $\bullet$ & & & $\bullet$ & \\
\hline 29. Ochlerotatus scapularis & & & & $\bullet$ & & $\bullet$ & & $\bullet$ & & & \\
\hline 30. Ochlerotatus serratus & $\bullet$ & & $\bullet$ & $\bullet$ & & $\bullet$ & & & $\bullet$ & & \\
\hline 31. Ochlerotatus terrens & $\bullet$ & & & & & & & & & & \\
\hline 32. Psorophora albigenu & & & & $\bullet$ & & $\bullet$ & & & & & \\
\hline 33. Psorophora ciliata & & & & $\bullet$ & & & & & & & \\
\hline 34. Psorophora confinnis & & & & $\bullet$ & & $\bullet$ & & & & & \\
\hline 35. Psorophora discrucians & & & & $\bullet$ & $\bullet$ & & & & & & \\
\hline 36. Psorophora ferox & $\bullet$ & & $\bullet$ & $\bullet$ & & $\bullet$ & & & $\bullet$ & & \\
\hline 37. Psorophora saeva & $\bullet$ & & & & & & & & & & \\
\hline 38. Psorophora varinervis & $\bullet$ & & & $\bullet$ & & $\bullet$ & & & $\bullet$ & & \\
\hline 39. Sabethes albiprivus & & & $\bullet$ & & & & & & & & \\
\hline 40. Sabethes aurescens & $\bullet$ & & $\bullet$ & & & & & & & & \\
\hline 41. Sabethes melanonymphe & & & & & & & & & & & \\
\hline 42. Sabethes purpureus & $\bullet$ & & & & & & & & $\bullet$ & & \\
\hline 43. Sabethes soperi & & & & & & & & & & & \\
\hline 44. Sabethes xyphydes & $\bullet$ & & & & & & & & & & \\
\hline 45. Toxorhynchites (Lynchiella) sp. & & & & • & & $\bullet$ & & & & & \\
\hline 46. Trichoprosopon compressum & $\bullet$ & & $\bullet$ & & & & & & & & \\
\hline 47. Trichoprosopon pallidiventer & $\bullet$ & & & & & $\bullet$ & & & $\bullet$ & & \\
\hline 48. Uranotaenia calosomata & & & & & & & & & & & \\
\hline 49. Uranotaenia geometrica & & & & $\bullet$ & & & & - & & & \\
\hline 50. Uranotaenia lowii & & & & $\bullet$ & & $\bullet$ & & & & & \\
\hline 51. Uranotaenia nataliae & & & $\bullet$ & $\bullet$ & & & & & & & \\
\hline 52. Wyeomyia codiocampa & & & & & & & & & & & \\
\hline 53. Wyeomyia davisi & & & $\bullet$ & $\bullet$ & & & & & $\bullet$ & & \\
\hline 54. Wyeomyia limai & $\bullet$ & & & & & & & & & & \\
\hline 55. Wyeomyia lopesi & & & & $\bullet$ & & & & & & & \\
\hline 56. Wyeomyia quasilongirostris & & & & $\bullet$ & & & & & & & \\
\hline 57. Wyeomyia serratoria & $\bullet$ & & & & & & & & & & \\
\hline
\end{tabular}


Anexo I. Distribuição das espécies de Aedes (Stegomyia) no Rio Grande do Sul, registradas de março de 1995 a abril de 2004 no SISFAD/FUNASA/

RS.

MUNICÍPIO

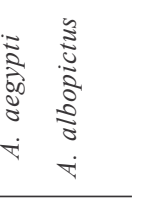

Agudo

Alecrim

Alegrete

Alegria

Alpestre

Alto Feliz

Alto Uruguai

Alvorada

Amaral Ferrador

Ametista do Sul

Anta Gorda

Antônio Prado

Arambaré

Araricá

Aratiba

Arroio do Meio

Arroio do Sal

Arroio dos Ratos

Bagé

Balneário Pinhal

Barra do Guarita

Barra do Ribeiro

Barra Funda

Barros Cassal

Bento Gonçalves

Boa Vista das Missões

Boa Vista do Buricá

Boa Vista do Cadeado

Boa Vista do Incra

Boa Vista do Sul

Bom Jesus

Bom Princípio

Bom Progresso

Bom Retiro do Sul

Bossoroca

Braga

Butiá

Cachoeira do Sul

Cachoeirinha

Caçapava do Sul

Cacequi

Cacique Doble

Caibaté

Caiçara

Camaquã

Campina das Missões

Campo Bom

Campo Novo

Campos Borges

Candelária

Cândido Godoi

Canela

Canguçu

Canoas

Canudos do Vale

Capão da Canoa

Capela de Santana
MUNICÍPIO

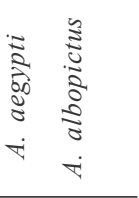

Capitão

Capivari do Sul

Caraá

Carazinho

Carlos Barbosa

Casca

Catuípe

Caxias do Sul

Cerro Branco

Cerro Grande

Cerro Grande do Sul

Cerro Largo

Chapada

Charqueadas

Cidreira

Colinas

Colorado

Condor

Constantina

Coqueiro Baixo

Coronel Barros

Coronel Bicaco

Cotiporã

Crissiumal

Cristal

Cristal do Sul

Cruz Alta

Cruzeiro do Sul

Derrubadas

Dezesseis de Novembro

Dilermando de Aguiar

Dois Irmãos

Dois Irmãos das Missões

Dom Feliciano

Dom Pedrito

Dom Pedro de Alcântara

Dr. Maurício Cardoso

Eldorado do Sul

Encantado

Encruzilhada do Sul

Engenho Velho

Entre-Ijuís

Erechim

Erval Seco

Esmeralda

Esperança do Sul

Espumoso

Estância Velha

Esteio

Estrela

Estrela Velha

Eugênio de Castro

Farroupilha

Fazenda Vila Nova

Feliz

Flores da Cunha

Formigueiro
MUNICÍPIO

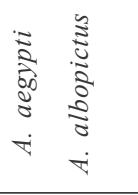

Forquetinha

Fortaleza dos Valos

Frederico Westphalen

Garibaldi

Garruchos

Gaurama

General Câmara

Giruá

Glorinha

Gramado

Gramado dos Loureiros

Gramado Xavier

Gravataí

Guabiju

Guaíba

Guaporé

Guarani das Missões

Horizontina

Humaitá

Ibarama

Ibirubá

Igrejinha

Ijuí

Imbé

Imigrante

Independência

Ipiranga do Sul

Iraí

Itaara

Itacurubi

Itaqui

Itati

Ivoti

Jaboticaba

Jacuizinho

Jaguarão

Jaguari

Jóia

Júlio de Castilhos

Lagoa Vermelha

Lajeado

Lajeado do Bugre

Liberato Salzano

Lindolfo Collor

Linha Nova

Machadinho

Mampituba

Manoel Viana

Maquiné

Maratá

Marau

Mariano Moro

Mata

Mato Queimado

Maximiliano de Almeida

Miraguaí

Montenegro
X

$\mathrm{X}$
$\mathrm{X} \quad \mathrm{X}$

$\mathrm{X}$

$\mathrm{X} \quad \mathrm{X}$

$\mathrm{X}$

$\mathrm{X}$
$\mathrm{X}$

$X$
$X$

$\mathrm{X}$

X

X

X $\mathrm{X}$

X X

X X

X X

X X

X

X X

X

X X

X X

$\mathrm{X}$
$\mathrm{X}$

$\mathrm{X} \quad \mathrm{X}$

X

$\mathrm{X}$

X

X X

$\mathrm{X}$

X

X

X

X

X

$\mathrm{X}$

X

X X

$\mathrm{X}$

X

$\mathrm{X}$

X X

X

$\mathrm{X}$

X

$X \quad X$

X

X X

$\mathrm{X}$

X

X

X

X

$\mathrm{X}$ 
Continuação Anexo I.

\begin{tabular}{|c|c|c|}
\hline MUNICÍPIO & $\begin{array}{l}\vec{z} \\
\tilde{D} \\
00 \\
8 \\
\dot{\nabla}\end{array}$ & 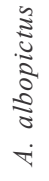 \\
\hline Morro Reuter & & $\mathrm{X}$ \\
\hline Morrinhos do Sul & & $\mathrm{X}$ \\
\hline Muçum & & $\mathrm{X}$ \\
\hline Não-Me-Toque & $\mathrm{X}$ & $\mathrm{X}$ \\
\hline Nonoai & & $\mathrm{X}$ \\
\hline Nova Bassano & & $\mathrm{X}$ \\
\hline Nova Boa Vista & & $\mathrm{X}$ \\
\hline Nova Candelária & $\mathrm{X}$ & $\mathrm{X}$ \\
\hline Nova Hartz & & $\mathrm{X}$ \\
\hline Nova Petrópolis & & $\mathrm{X}$ \\
\hline Nova Prata & & $\mathrm{X}$ \\
\hline Nova Ramada & & $\mathrm{X}$ \\
\hline Nova Santa Rita & & $\mathrm{X}$ \\
\hline Novo Barreiro & & $\mathrm{X}$ \\
\hline Novo Cabrais & & $\mathrm{X}$ \\
\hline Novo Hamburgo & $\mathrm{X}$ & $\mathrm{X}$ \\
\hline Novo Machado & $\mathrm{X}$ & $\mathrm{X}$ \\
\hline Novo Tiradentes & & $\mathrm{X}$ \\
\hline Novo Xingú & & $\mathrm{X}$ \\
\hline Osório & & $\mathrm{X}$ \\
\hline Paim Filho & & $\mathrm{X}$ \\
\hline Palmares do Sul & & $\mathrm{X}$ \\
\hline Palmeira das Missões & $\mathrm{X}$ & $\mathrm{X}$ \\
\hline Palmitinho & $\mathrm{X}$ & $\mathrm{X}$ \\
\hline Panambi & & $\mathrm{X}$ \\
\hline Pantano Grande & & $\mathrm{X}$ \\
\hline Paraíso do Sul & & $\mathrm{X}$ \\
\hline Pareci Novo & & $\mathrm{X}$ \\
\hline Parobé & & $\mathrm{X}$ \\
\hline Passo do Sobrado & & $\mathrm{X}$ \\
\hline Passo Fundo & & $\mathrm{X}$ \\
\hline Paverama & & $\mathrm{X}$ \\
\hline Pelotas & $\mathrm{X}$ & $\mathrm{X}$ \\
\hline Picada Café & $\mathrm{X}$ & $\mathrm{X}$ \\
\hline Pinhal & & $\mathrm{X}$ \\
\hline Pinheirinho do Vale & $\mathrm{X}$ & $\mathrm{X}$ \\
\hline Pinheiro Machado & $\mathrm{X}$ & $\mathrm{X}$ \\
\hline Pirapó & $\mathrm{X}$ & \\
\hline Planalto & & $\mathrm{X}$ \\
\hline Poço das Antas & & $\mathrm{X}$ \\
\hline Portão & & $\mathrm{X}$ \\
\hline Porto Alegre & $\mathrm{X}$ & $\mathrm{X}$ \\
\hline Porto Lucena & $\mathrm{X}$ & $\mathrm{X}$ \\
\hline Porto Mauá & $\mathrm{X}$ & $\mathrm{X}$ \\
\hline Porto Vera Cruz & $\mathrm{X}$ & \\
\hline Porto Xavier & $\mathrm{X}$ & $\mathrm{X}$ \\
\hline Presidente Lucena & & $\mathrm{X}$ \\
\hline Quaraí & & $\mathrm{X}$ \\
\hline Quinze de Novembro & & $\mathrm{X}$ \\
\hline Redentora & & $\mathrm{X}$ \\
\hline Restinga Seca & & $\mathrm{X}$ \\
\hline Rio dos Índios & & $\mathrm{X}$ \\
\hline Rio Grande & $\mathrm{X}$ & $\mathrm{X}$ \\
\hline Rio Pardo & $\mathrm{X}$ & $\mathrm{X}$ \\
\hline Riozinho & & $\mathrm{X}$ \\
\hline Roca Sales & & $\mathrm{X}$ \\
\hline Rodeio Bonito & & $\mathrm{X}$ \\
\hline Rolador & & $\mathrm{X}$ \\
\hline
\end{tabular}

MUNICÍPIO

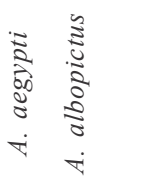

Rolante

Ronda Alta

Rondinha

Roque Gonzales

Rosário do Sul

Sagrada Família

Salto do Jacuí

Salvador das Missões

Salvador do Sul

Sananduva

Santa Bárbara do Sul

Santa Clara do Sul

Santa Cruz do Sul

Santa Maria

Santa Maria do Herval

Santa Rosa

Santa Tereza

Santana do Livramento

Santiago

Santo Ângelo

Santo Antônio da Patrulha

Santo Antônio das Missões

Santo Antônio do Planalto

Santo Augusto

Santo Cristo

Santo Expedito do Sul

São Borja

São Francisco de Assis

São Francisco de Paula

São Gabriel

São Jerônimo

São José das Missões

São José do Hortêncio

São José do Inhacorá

São José do Polêsine

São José do Sul

São Leopoldo

São Luiz Gonzaga

São Marcos

São Martinho

São Martinho da Serra

São Miguel das Missões

São Nicolau

São Paulo das Missões

São Pedro da Serra

São Pedro das Missões

São Pedro do Butiá

São Pedro do Sul

São Sebastião do Caí

São Sepé

São Valentin do Sul

São Valério do Sul

São Vendelino

São Vicente do Sul

Sapiranga

Sapucaia do Sul

Sarandi

Seberi

$\mathrm{X} \quad \mathrm{X}$

$\mathrm{X} \quad \mathrm{X}$

$\mathrm{X}$
MUNICÍPIO

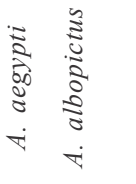

Sede Nova

X

Segredo

Selbach

Senador Salgado Filho

Serafina Corrêa

Sério

Sete de Setembro

Sinimbu

Sobradinho

Tabaí

Tapejara

Tapera

Tapes

Taquara

Taquari

Taquaruçu do Sul

Tenente Portela

Terra de Areia

Teutônia

Tio Hugo

Tiradentes do Sul

Toropi

Torres

Tramandaí

Travesseiro

Três Cachoeiras

Três Coroas

Três de Maio

Três Forquilhas

Três Palmeiras

Três Passos

Trindade do Sul

Triunfo

Tucunduva

Tunas

Tupandi

Tuparendi

Ubiretama

Unistalda

Uruguaiana

Vacaria

Vale do Sol

Vale Real

Vale Verde

Venâncio Aires

Vera Cruz

Veranópolis

Viadutos

Viamão

Victor Graeff

Vicente Dutra

Vila Maria

Vista Alegre

Vista Alegre do Prata

Vista Gaúcha

Vitória das Missões

Westfália

Xangrilá

$\mathrm{X}$

X

X X

X

X

X

X

X

$\mathrm{X}$

X X

$\mathrm{X} X$

X

X

$\mathrm{X}$
$\mathrm{X}$
$\mathrm{X}$

X X

$\mathrm{X} \quad \mathrm{X}$

X X

$X$

$\mathrm{X} \quad \mathrm{X}$

X X

X X

$X$

$\mathrm{X} \quad \mathrm{X}$

X

X X

$X$

X X

X X

$\mathrm{X}$
$\mathrm{X}$

X X

X

$\mathrm{X}$
$\mathrm{X}$

$\mathrm{X}$
$\mathrm{X}$

X

X

X

X

X

X

$\mathrm{X}$

X X

X

X

X X

X X

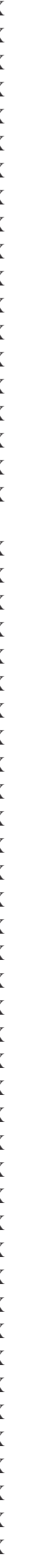


desta informação é verificada no trabalho de Löwenberg-Neto \& Silva (2002), onde, ao registrarem Ae. albopictus para Santa Catarina, afirmaram que, dentre os estados do sul do Brasil, esta espécie ocorria apenas no Paraná. Esta lacuna foi sanada com o trabalho de Santos (2003) que atualizou a distribuição Ae. albopictus no Brasil, citando-a para o RS. As espécies $C x$. quinquefasciatus e Oc. crinifer, provavelmente ocorram em todas as zonas fisiográficas, já que possuem ampla distribuição, conforme aponta Forattini (2002) para as duas primeiras e Guimarães (1997) para Oc. crinifer.

Por outro lado, Cq. fasciolata, Cq. shannoni, Cx. corniger, Cx. imitator, Cx. saltanensis, Li. durhami, Li. flavisetosus, Ma. humeralis, Ma. pseudotitillans, Ma. wilsoni, Oc. terrens, Ps. ciliata, Ps. saeva, Sa. melanonymphe, Sa. purpureus, Sa. soperi, Sa. xyphydes, Tr. compressum, Ur. calosomata, Ur. geometrica, Ur. nataliae, Wy. codiocampa, Wy. davisi, Wy. lopesi, Wy. quasilongirostris e Wy. serratoria são apontadas em apenas uma zona fisiográfica (Tabela I). Estes poucos registros sugerem a necessidade de realização de novas coletas para melhor avaliar a real distribuição geográfica destes culicíneos no Rio Grande do Sul.

Os registros de Ochlerotatus condolescens Dyar \& Knab, como Aedes condolensis, feito por Vasconcelos et al. (2003) e Culex inflictus Theobald, por Ruas-Neto et al. (1994), diferem das informações de distribuição contidas em Guimarães (1997). Para esse autor, Oc. condolescens ocorre nas Bahamas, Cuba, Ilhas Cayman e Peru. No que se refere a $C x$. inflictus, compreende o México e alguns países da América Central e norte da América do Sul, sendo na Venezuela, a ocorrência de maior latitude sul. Além disso, não foram verificados exemplares em coleções, o que nos leva a deixá-las fora da lista até que a ocorrência destas espécies, no Estado, seja esclarecida.

No que diz respeito aos aspectos epidemiológicos associados aos mosquitos, Primio (1937) já alertava para a possibilidade de doenças como dengue e febre amarela, nas formas urbana e silvestre, se instalarem no Estado. Forattini (1999), aponta os dois fatores responsáveis para o aparecimento de endemia urbana de febre amarela: presença do vetor e circulação do vírus. Sendo assim, Ae. aegypti é reconhecido pelo seu papel vetorial, tanto de dengue quanto de febre amarela urbana, além de estar amplamente distribuído no RS. Por outro lado, Vasconcelos et al. (2003), isolaram o vírus amarílico em exemplares de $\mathrm{Hg}$. leucocelaenus coletados em áreas silvestres dos municípios de Garruchos e Santo Antônio das Missões, fato que motivou o Ministério da Saúde a classificar o RS como área de transição para febre amarela, onde o vírus circula no ambiente silvestre, podendo acometer o homem em surtos esporádicos. Diante desse quadro, Ae. albopictus aparece como uma espécie de ligação, já que freqüenta ambientes silvestres, rurais, periurbanos e urbanos, podendo conectar os ciclos silvestre e urbano da febre amarela (Consoli \& Lourenço-de-Oliveira 1994).

Sendo assim, várias espécies citadas necessitam de atenção especial por parte dos órgãos de saúde em virtude dos parasitas por elas transmitidos, principalmente, Ae. aegypti, Ae. albopictus e Hg. leucocelaenus. Estudos abordando aspectos de bio-ecologia devem ser levados a efeito para melhor conhecermos as relações dessas espécies com seus habitats e hospedeiros vertebrados.

Agradecimentos. Ao estudante de Biologia da PUCRS Jorge Alberto Silveira Suñé, pela indispensável contribuição nas coletas. Aos pesquisadores Dr. Ronaldo Toma (MZSP) e Dr. Sebastião José de Oliveira (IOC) pelas informações sobre seus acervos. À médica-veterinária Dóris Bercht Brack da Divisão de Vigilância Ambiental em Saúde (DVAS/RS) e ao servidor da FUNASA/RS Carlos Francisco Ferreira, pelas informações sobre os registros de Aedes spp. no RS. Aos biólogos Fernando R. Meyer (MAPA), Fernanda de Mello, da Seção de Reservatórios e Vetores (LCRS), e Hilda Alice de Oliveira Gastal (MCNZ), por possibilitarem o exame das coleções. À Prof. Dra. Maria Anice Mureb Sallum (FSP/USP), pela presteza na confirmação das identificações e valiosas sugestões para o trabalho.

\section{REFERÊNCIAS}

Arend, L. M. 1997. Geografia Física, p. 22-53. In: G. R. Hoffmann, L. M. Arend; J. C. B. da Silveira; H. R. Bellomo \& J. L. M. Nunes (eds). Rio Grande do Sul: Aspectos da Geografia 4. ed. Porto Alegre, Martins Livreiro, 104p.

Cardoso, J. da C.; E. Corseuil \& J. M. S. Barata. 2004. Anophelinae (Diptera, Culicidae) ocorrentes no estado do Rio Grande do Sul, Brasil. Entomología y Vectores 11:159-177.

Consoli, R. A. G. B. \& R. Lourenço-de-Oliveira. 1994. Principais mosquitos de importância sanitária no Brasil. Rio de Janeiro, Editora FIOCRUZ, 225p.

Correa, R. R. \& G. R. Ramalho. 1956. Revisão de Phoniomyia Theobald, 1903 (Diptera, Culicidae, Sabethini). Folia Clinica et Biologica 25: $1-176$.

Darsie-Jr, R. F. 1985. Mosquitoes of Argentina. Part I. Keys for identification of adult females and fourth stage larvae in English and Spanish (Diptera, Culicidae). Mosquito Systematics 17: 153253.

Forattini, O. P. 1962. Entomologia médica: parte geral, Diptera, Anophelini. v. 1. São Paulo, Faculdade de Saúde Pública/USP, $662 p$.

Forattini, O. P. 1965a. Entomologia médica: Culicini: Culex, Aedes e Psorophora. v. 2. São Paulo, Editora da Universidade de São Paulo, 506p.

Forattini, O. P. 1965b. Entomologia médica: Culicini: Haemagogus, Mansonia, Culiseta. Sabethini. Toxorhynchitini. Arboviroses. Filariose bancroftiana. Genética. v. 3. São Paulo, Editora da Universidade de São Paulo, $416 \mathrm{p}$.

Forattini, O. P. 1999. Febre amarela. Revista de Saúde Pública 33:534.

Forattini, O. P. 2002. Culicidologia médica: identificação, biologia, epidemiologia. v. 2. São Paulo, Editora da Universidade de São Paulo, 860p.

Fundação Nacional de Saúde. Coordenação Regional do Rio Grande do Sul (FUNASA/RS). Banco de Dados do Programa de Controle da Febre amarela e Dengue (PCFAD). Consulta em: 20 março 2003

Guimarães, J. H. 1997. Systematic database of Diptera of the Americas South of the United States (family culicidae). São Paulo, Plêiade/FAPESP, 286p.

Harbach, R. E. \& I. J. Kitching. 1998. Phylogeny and classification of the Culicidae (Diptera). Systematic Entomology 23: 327-370.

Harbach, R. E. \& E. L. Peyton. 2000. Sistematics of Onirion, a new genus of Sabethini (Diptera: Culicidae) from the Neotropical Region. Bulletin of the Natural History Museum (Entomology Series) 69: 115-169.

Hutchings, R. S. G.; M. A. M. Sallum \& R. L. M. Ferreira. 2002. Culicidae (Diptera: Culicomorpha) da Amazônia Ocidental Brasileira: Querari. 
Acta Amazonica 32: 109-122.

Judd, D. D. 1998. Review of a bromeliad-ovipositing lineage in Wyeomyia and the resurrection of Hystatomyia (Diptera: Culicidae). Annals of the Entomological Society of America 91: 572-589.

Lane, J. 1953. Neotropical Culicidae. 2. v. São Paulo, Universidade de São Paulo, 1112 p.

Lane, J. \& N. L. Cerqueira. 1942. Os Sabetíneos da América (Diptera: Culicidae). Arquivos de Zoologia do Estado de São Paulo 3: 473-849.

Löwenberg-Neto, P. \& M. A. N. da Silva. 2002. Primeiro registro de Aedes albopictus no Estado de Santa Catarina, Brasil. Revista de Saúde Pública 36: 246-247.

Lutz, A.; H. C. de S. Araujo \& O. da Fonseca-Filho. 1918. Viagem scientifica no Rio Paraná e a Assuncion com volta por Buenos Aires, Montevideo e Rio Grande. Memórias do Instituto Oswaldo Cruz 10: 104-171.

Marcondes, C. B.; A. Fernandes; U. Paterno; G. A. Müller; L. C. de Pinho \& D. V. Struffaldi. 2003. New records of mosquitoes from the southern Brazilian States of Santa Catarina and Rio Grande do Sul, with 18 species new for the States (Diptera: Culicidae). Zootaxa 347: $1-6$.

Netto, A. S. 1940. Mosquitos do Rio Grande do Sul. Porto Alegre, Faculdade de Medicina de Porto Alegre, 101p.

Papavero, N. \& J. H. Guimarães. 2000. The taxonomy of brasilian insects vectors of transmissible diseases $(1900-2000)$ - then and now. Memórias do Instituto Oswaldo Cruz 95: 109-118.

Peryassú, A. G. 1908. Os culicideos do Brazil. Rio de Janeiro, Typographia Leuzinger, 407p.

Pinto, C. 1932. Alguns mosquitos do Brasil e do oriente da Bolívia (Diptera. Culicidae). Revista Medico-Cirurgica do Brasil: 285309.

Pinto, C.; A. S. Netto \& H. Marques. 1940. Mosquitos do Rio Grande do Sul. Arquivos do Departamento Estadual de Saúde do Rio Grande do Sul 1: 14-51.

Primio, R. Di. 1935. Alguns culicídeos do Rio Grande do Sul. considerações nosológicas a respeito. Arquivos Riograndenses de Medicina 4: $127-164$.

Primio, R. Di. 1937. Em torno de alguns transmissores de doenças no Rio Grande do Sul. Arquivos Riograndenses de Medicina 7: 305-314.
Reinert, J. F. 1999. Restoration of Verrallina to generic rank in tribe Aedini (Diptera: Culicidae) and descriptions of the genus and three included subgenera. Contributions of the American Entomological Institute 31: 1-83.

Reinert, J. F. 2000a. New classification for the composite genus Aedes (Diptera: Culicidae: Aedini), elevation of subgenus Ochlerotatus to generic rank, reclassification of the other subgenera, and notes on certain subgenera and species. Journal of the American Mosquito Control Association 16: 175-188.

Reinert, J. F. 2000b. Restoration of Ayurakitia to generic rank in tribe Aedini and a revised definition of the genus. Contributions of the American Entomological Institute 16: 57-65.

Ronna, E. 1924. Apontamentos da microfauna Rio-Grandense. Egatea 9: $267-272$.

Ruas-Neto, A. L. \& S. M. Silveira. 1989. Uso de inseticidas bacterianas para o controle de culicídeos e simulídeos no Rio Grande do Sul. Memórias do Instituto Oswaldo Cruz 84: 39-45.

Ruas-Neto, A. L.; S. M. Silveira \& E. R. da C. Colares. 1994. Mosquito control based on larvicides in the state of Rio Grande do Sul, Brazil choice of the control agent. Cadernos de Saúde Pública 10: $222-230$.

Santos, R. La C. 2003. Atualização da distribuição de Aedes albopictus no Brasil (1997-2002). Revista de Saúde Pública 37: 671-673.

Stone, A. 1967. Notes on Psorophora lineata (Humboldt) and P. saeva Dyar \& Knab (Diptera, Culicidae). Revista Brasileira de Entomologia 12: 59-65.

Vasconcelos, P. F. C.; A. F. Sperb; H. A. O. Monteiro; M. A. N. Torres; M. R. S. Souza; H. B. Vasconcelos; L. B. L. F. Mardini \& S. G. Rodrigues. 2003. Isolations of yellow fever virus from Haemagogus leucocelaenus in Rio Grande do Sul State, Brazil. Transactions of the Royal Society of Tropical Medicine and Hygiene 97: 6062 .

Vianna, E. E. S.; P. R. P. Costa \& P. B. Ribeiro. 1996a. Oviposição e longevidade de adultos do Culex quinquefasciatus Say, 1823 (Diptera: Culicidae) em condições ambientais, em Pelotas, RS. Revista Brasileira de Parasitologia Veterinária 5: 47-52.

Vianna, E. E. S.; P. R. P. Costa \& P. B. Ribeiro. 1996b. Longevidade e viabilidade do ciclo aquático do Culex quinquefasciatus Say, 1823 (Diptera: Culicidae) em condições ambientais, em Pelotas, RS Revista Brasileira de Parasitologia Veterinária 5: 53-56. 